\title{
Laparoscopic inguinal hernia repair and Lichtenstein tension-free hernia repair for children in 13-18 years old: a prospective, randomized, single-blind controlled trial.
}

\author{
Zhengmin Cui ${ }^{1}$, Beibei Xie ${ }^{2}$, Rongpeng Zhang ${ }^{*}$ \\ ${ }^{1}$ Department of Pediatric Surgery, Linyi People's Hospital, Shandong University, 27 Jiefang Road, Linyi, PR China \\ ${ }^{2}$ Department of Gynecology, Linyi People's Hospital, Shandong University, 27 Jiefang Road, Linyi, PR China
}

\begin{abstract}
To compare the safety, feasibility, advantages and limits of laparoscopic herniorrhaphy and Lichtenstein technique for inguinal hernia repair. A total of 506 patients between 13 and $18 \mathrm{y}$ old were enrolled in this study and divided into three groups to receive transabdominal preperitoneal hernia repair (TAPP) $(n=168)$, total extraperitoneal hernia repair (TEP) $(n=168)$ or Lichtenstein repair $(n=170)$, respectively. Results suggested that the postoperative pain scores in the TAPP and TEP groups were lower than those in the Lichtenstein group, but the hospitalization costs of the TAPP and TEP groups were higher than those in the Lichtenstein group. None of the patients from TAPP and TEP groups showed recurrence, while 1 of the 170 patients from the Lichtenstein group showed recurrent hernia. It is indicated that laparoscopic herniorrhaphy including TAPP and TEP is safe and feasible, and it is superior to Lichtenstein technique in improving postoperative pain and avoiding recurrence, but it is more expensive than the latter.
\end{abstract}

Keywords: Laparoscopic inguinal hernia repair, Lichtenstein tension-free hernia repair, TAPP, TEP, RCT.

Accepted on November 2, 2017

\section{Introduction}

Nowadays, more and more patients underwent laparoscopic inguinal hernia repair surgery [1-3], but results and efficacy of laparoscopic hernia repair are still controversial [4-6] in 13-18 y old young people. In 1989, Lichtenstein et al. [6] first reported and used inguinal hernia repairs in the tension-free repair area. With the development of minimally invasive surgery, laparoscopic surgery has been widely applied and developed in hernia surgery. At present, Laparoscopic Inguinal Hernia Repair (LIHR) mainly includes two kinds Transabdominal Preperitoneal (TAPP) [7] hernia repair and Total Extraperitoneal (TEP) hernia repair [8].

Although the repair principle of TAPP and TEP is basically the same, their inside pre-periaural clearance of the path is different. TAPP technique is through the abdominal cavity into the peritoneal space, which does not need to pass through the abdominal cavity, it was generally considered not easy to produce intra-abdominal complications [9]. In the present study, we used a prospective randomized study to compare TAPP, TEP and Lichtenstein tension-free hernioplasty in terms of operative time, postoperative hospital stay, surgical complications, postoperative pain scores, and postoperative hernia recurrence rates and to further evaluate the safety, feasibility and advantages and disadvantages of laparoscopic inguinal hernia repair.

\section{Materials and Methods}

\section{General social demographic characteristics}

From June 2009 to October 2016, a total of 506 patients between 13 and 18 y old were enrolled in this study. They were randomly divided into three groups to receive TAPP $(n=168)$, TEP $(n=168)$ or Lichtenstein repair $(n=170)$, respectively. All patients were informed of the risks and differences between three surgical methods before surgery, and signed a written informed consent form. All recruited patients were followed up for three years. This study was approved by the Hospital Ethics Committee.

We collected the data including the age, sex, hernia types (oblique hernia, straight hernia and femoral hernia), herniasub types (typesI-IV), operation time, postoperative pain scores, hospitalization costs and time, postoperative scrotal seroma or esthesiodermia, postoperative local sensory disturbance, postoperative chronic pain and postoperative recurrent hernia for each patient.

\section{Case selection and exclusion criteria}

The criteria for selection were inguinal hernia patients at age of 13-18 y old who were able to tolerate pneumoperitoneum surgery. Exclusion criteria mainly included heart, liver and 
lung dysfunction, and no toleration in pneumoperitoneum surgery.

\section{Lichtenstein surgical method}

This Lichtenstein surgical method was conducted by surgeon Zhang. The patients were referred to persistent epidural anesthesia. The anatomy of the skin incision and inguinal wall was the same as the Bassini repair method. During the procedure, the hernia sacs of the patients were completely divested. If the hernia sacs in the patients were larger, the hernia sac closer side would be cut off, and closed from the inner ring and then back to the abdominal cavity, and then in the neck of the hernia sac bag for suture. For further spermatic cord dissociation, $6 \mathrm{~cm} \times 11 \mathrm{~cm}$ of the United States Johnson \& Johnson WeibuII patch was covered in the abdominal transverse fascia and spermatic cord before intra-abdominal oblique, combined tendon, pubic bone periosteum and groin ligaments using a polypropylene mesh and fixed using nylon 2-0. Spermatic cord through the gap could not be too tight, avoiding the ischemic atrophy of testis.

\section{TAPP surgical method}

TAPP technology was conducted by surgeon Wang in our hospital according to the procedure of Dion et al. [10] using tracheal intubation intravenous anesthesia. The pneumoperitoneal pressure was 9 to $12 \mathrm{~mm} \mathrm{Hg}(1 \mathrm{~mm}$ $\mathrm{Hg}=0.133 \mathrm{kPa}$ ) in this method. A $10 \mathrm{~mm}$ trocar was placed on the lower edge of the umbilical hole as an observation hole. A $5 \mathrm{~mm}$ trocar was placed on the outer edge of the rectus abdominis between the contralateral umbilical and the anterior superior iliac spine. Under the laparoscopy, the hernia sac was pulled into the abdominal cavity through the sharp separation of the peritoneal space. From the edge of the hernia ring edge of the abdominal wall to the surrounding free peritoneum, the abdominal wall blood vessels, combined tendon, abdominal transverse arch, spermatic cord and inguinal ligament, pubic comb ligament were dissected. The patch from the iliac crest to the common tendon, to the pubic symphysis, to the pubic ligament, and to $10 \mathrm{~mm}$ trocar was built into the $12 \times 15 \mathrm{~cm}$ American Johnson violet II patch which was fixed on the Cooper ligament. The ventral fascia, the iliac shiver and the sutured flap were closed with endoscopic suture nail (ENDOPATH EMS). The TAPP surgery of bilateral hernia was treated in the same way.

\section{TEP surgical method}

TEP technique was conducted by surgeon Tang in our hospital using tracheal intubation intravenous anesthesia. The surgeon and his assistants were standing on the contralateral side of the patients. Patients lay down in supine position, and their heads were $15-30^{\circ}$ lower than feet. A $2 \mathrm{~cm}$ small incision was cut along the abdominal white line, exposing the posterior rectus sheath along the rectus abdominis after the separation of 10 $\mathrm{mm}$ trocar. Subsequently, the peritoneal space was acutely separated between the loose tissue, pubic symphysis and bilateral pubic comb ligament were exposed, spermatic cord and hernia sac could be observed. The hernia sac and spermatic cord were then completely separated, and made "Spermatic cord component abdominal wall", which were exposed to vas deferens, spermatic cord blood vessels and "dangerous triangle". A $10 \times 15 \mathrm{~cm}$ of the United States Johnson \& Johnson WeibuII patch must cover the straight hernia triangle, oblique hernia mouth and must be lateral to the iliac before the upper ridge. To invoid the damage of the clamp to suppress the two lower corners of the patch, the formation of patches and the peritoneum close to the patch must be ensured. The surgery of bilateral hernia was done in accordance with the same method.

\section{Postoperative treatment and observation}

During intraoperative and postoperative, patients were given routine rehydration and prophylactic use of antibiotics, semiliquid diet after $6 \mathrm{~h}$ of anesthesia, and food after $1 \mathrm{~d}$ of surgery. The operative time, postoperative hospital stay, complications, postoperative pain scores, postoperative analgesic drugs, postoperative local skin sensory abnormalities, postoperative chronic pain and recurrence of postoperative long-term hernia were recorded and compared. The postoperative pain scores were calculated based on Numerical Rating (NRS).

\section{Statistics out and analysis methods}

We used SPSS 23.0 software to analyse the data. Quantitative data was shown as mean \pm Standard Deviation (SD). The differences between the three groups were analysed by variance analysis or chi-square analysis. The difference $\mathrm{P}<0.05$ between the groups was statistically significant.

\section{Results}

\section{Analysis of general demographic characteristics}

Results in Table 1 showed that there were no statistically significant differences in the general demographic characteristics between the three groups $(\mathrm{P}>0.05)$.

Table 1. General demographic characteristics.

\begin{tabular}{lllllll}
\hline Group & Age $(y)$ & \multicolumn{2}{c}{ Gender } & & Hernia type & Hernia subtype \\
\cline { 3 - 6 } & & Male & Female & $\begin{array}{l}\text { Oblique } \\
\text { hernia }\end{array}$ & $\begin{array}{l}\text { Straight } \\
\text { hernia }\end{array}$ & $\begin{array}{l}\text { Femoral } \\
\text { hernia }\end{array}$ I type II type III type IV type \\
\hline
\end{tabular}


Laparoscopic inguinal hernia repair and Lichtenstein tension-free hernia repair for children in 13-18 years old: a prospective, randomized, single-blind controlled trial

\begin{tabular}{|c|c|c|c|c|c|c|c|c|c|c|}
\hline TAPP group $(n=168)$ & $14.83 \pm 1.75$ & 141 & 27 & 150 & 14 & 4 & 21 & 124 & 18 & 5 \\
\hline TEP group $(n=168)$ & $14.84 \pm 1.45$ & 134 & 34 & 147 & 16 & 5 & 23 & 119 & 20 & 6 \\
\hline Lichtenstein group $(n=170)$ & $15.18 \pm 1.55$ & 132 & 38 & 145 & 18 & 7 & 23 & 124 & 19 & 4 \\
\hline $\mathrm{F}$ value/Chi-square value & 2.542 & 2.191 & & 1.441 & & & & & & \\
\hline$P$ value & 0.08 & 0.334 & & 0.837 & & & 0.99 & & & \\
\hline
\end{tabular}

\section{Comparison of the results in three groups under surgery}

The results of the comparison between the three groups were shown in Table 2. The procedures were completed in all the patients of the three groups without conversion to open surgery. Postoperative follow-up was 16 months (range 5 to 34 months). Results measured by Bonferroni methods showed that the postoperative pain scores in the TAPP and TEP groups were significantly lower than that in the Lichtenstein group $(2.75 \pm 0.89$ and $1.78 \pm 0.57$ vs. $4.23 \pm 0.49, \mathrm{P}<0.05)$, but the hospitalization costs of the TAPP and TEP groups were significantly higher than that in the Lichtenstein group (13003 $\pm 815 \mathrm{RMB}$ and $11962 \pm 914 \mathrm{RMB} v s .7072 \pm 448 \mathrm{RMB}$, $\mathrm{P}<0.05)$. None patients from the TAPP and TEP groups had recurrence, while 1 of the 170 patients from the Lichtenstein group showed recurrent hernia $(0.59 \%, \mathrm{P}>0.05)$. Additionally, no significant difference was observed among the three groups in operation time, postoperative scrotal seroma or esthesiodermia, local sensory disturbance and chronic pain $(\mathrm{P}>0.05)$.

Table 2. Comparison of the three groups' indicators.

\begin{tabular}{|c|c|c|c|c|c|c|c|}
\hline Group & Operation time & $\begin{array}{l}\text { Postoperative pain } \\
\text { scores }\end{array}$ & $\begin{array}{l}\text { Hospitalization } \\
\text { costs }\end{array}$ & $\begin{array}{l}\text { Postoperative } \\
\text { seroma } \\
\text { esthesiodermia }\end{array}$ & $\begin{array}{l}\text { Postoperativ } \\
\text { e local } \\
\text { sensory } \\
\text { disturbance }\end{array}$ & $\begin{array}{l}\text { Postoperative } \\
\text { chronic pain }\end{array}$ & $\begin{array}{l}\text { Postoperativ } \\
\text { e recurrent } \\
\text { hernia }\end{array}$ \\
\hline TAPP group $(n=168)$ & $48.42 \pm 13.81$ & $2.75 \pm 0.89^{\#}$ & $13003.21 \pm 814.8^{\#}$ & $23(13.7 \%)$ & $15(8.9 \%)$ & $2(1.2 \%)$ & 0 \\
\hline TEP group $(n=168)$ & $50.87 \pm 16.20$ & $1.78 \pm 0.57^{\#}$ & $11962.40 \pm 913.97^{\#}$ & $25(14.9 \%)$ & $17(10.1 \%)$ & $3(1.8 \%)$ & 0 \\
\hline $\begin{array}{l}\text { Lichtenstein } \quad \text { group } \\
(\mathrm{n}=170)\end{array}$ & $48.78 \pm 15.60$ & $4.23 \pm 0.49$ & $7072.18 \pm 448.01$ & $15(8.8 \%)$ & $18(10.6 \%)$ & $5(2.9 \%)$ & $1(0.59 \%)$ \\
\hline $\begin{array}{l}\mathrm{F} \text { value/Chi-square } \\
\text { value }\end{array}$ & 1.272 & 566.08 & 3003.795 & 3.199 & 0.277 & $0.505^{*}$ & $<0.01^{*}$ \\
\hline$P$ value & 0.281 & $<0.01$ & $<0.01$ & 0.202 & 0.871 & & \\
\hline
\end{tabular}

*Fisher exact probability method; \#compared with Lichtenstein group and $\mathrm{P}<0.01$, using Bonferroni methods

\section{Discussion}

Lichtenstein et al. [6] advocated the use of synthetic materials to strengthen the posterior wall of the inguinal canal "tensionfree hernia repair". At present, tension-free repair methods such as Rutkow repair, Lichtenstein repair, TAPP, TEP and so on are commonly used to treat inguinal hernia. Studies have shown that the recurrence rate of tension-free hernia repair in the primary hernia is only $0.1 \%$, and the recurrence of hernia was $2 \%$. Our study suggested that TAPP and TEP groups had no recurrence and the recurrence rate of Lichtenstein group was $0.59 \%$.

The results of this study suggested that, compared with the Lichtenstein group, TAPP group, TEP group had a small incision, light pain postoperative, lower extremity venous thrombosis, and reduced bed rest Pneumonia and other complications. Moreover, operation time showed no significant difference between TAPP group, TEP group and Lichtenstein group. The long-term recurrence rate of TAPP group and TEP group was lower than the tension-free repair surgery, due to laparoscopic hernia repair patch into a larger area. If open hernia repair was also placed in the same size patch and the recurrence rate was to be further confirmed. However, open surgery to place the same size of the patch must have greater incision and groin area anatomy, and was not conducive to patients with postoperative recovery. Furthermore, large surgical incision easily led to surgical incision infection and local skin and nerve vascular injury, and local skin sensory dysfunction. Hemorrhage is the most common complication after LIHR surgery. Previous study reported that the incidence of hemorrhage after LIHR surgery was 1.9-22.9\% [11], and this study showed that the incidence of hemorrhage in TAPP group and TEP group were 13.1\% (11/84) and 15.5\% (13/84), respectively which was mainly due to the accumulation of residual fluid in the distal hernia sac after the sacrificing hernia sac, resulting in the secretion of liquid or hernia contents. A longer history of the adhesion of the hernia sac or huge scrotal hernia resection rate was higher. Patients with oblique hernia, such as the complete removal of the hernia sac could reduce the occurrence of serum swollen, but the adhesion of dense 
hernia sac might be caused by the occurrence of hematoma. The general serum swollen to clarify the light yellow liquid, 1 $\mathrm{w}$ after the start of their own absorption, mostly in 1-2 months could subside [12]. The patients needed for daily heat treatment to speed up the absorption. In addition to special circumstances, the patients did not need surgery intervention. The real hematoma is the groin area or the scrotal sulcus. Intraoperative vascular injury or bleeding was the main reason for the occurrence of postoperative hematoma. Young patients had high vascular fragility and wound bleeding of them could also cause hematoma. Most of the thick hematoma was not easy to puncture and physical therapy comprehensive treatment. Patients must be careful to avoid the recurrence of serum and unnecessary surgery through the B-ultrasound.

This study also showed that the complications between the laparoscopic hernia repair and open surgery had no significant difference, but the hernia and bilateral TAPP hernia repair had unique advantages. LIHR surgical indications could be summarized into four categories of people: pre-retroperitoneal indications of the crowd; needing to resume activities and work as soon as possible; recurrent hernia and bilateral hernia; and special needs and wishes of patients. In addition, the conditions of the hospital and the laparoscopic technique and experience of the surgeons were also important factors that must be considered. Otherwise it might produce unnecessary recurrence and complications $[13,14]$. This study indicated that the cost of surgery in TEP group and TAPP group was significantly higher than that in Lichtenstein group. Laparoscopic instruments are necessary for LIHR which can be used in conditional hospitals to make full use of existing resources without additional cost. In addition to the patch without the use of other consumables, the use of reusable laparoscopic instruments instead of disposable devices can reduce the cost of surgery. Also, previous study reported that patch fixation had nothing to do with the recurrence rate, so that no other fixed patch [15]. Hernia defect suture bigger than $4 \mathrm{~cm}$ instead of nail fixation patch could be used to avoid the use of stapled material and greatly reduce the cost of surgery. LIHR surgery using laparoscopic resources in the hospital increased the cost of laparoscopy and anesthesia, but the integrated LIHR had advantages such as quick postoperative recovery, less pain and better repair. The increased cost is acceptable for most patients. It is believed that, with the development of laparoscopic technology, laparoscopic hernia repair will be widely accepted and recognized.

\section{Acknowledgements}

None

\section{Conflicts of Interest}

None

\section{References}

1. Yang XF, Liu JL. Anatomy essentials for laparoscopic inguinal hernia repair. Ann Transl Med 2016; 4: 372.
2. Yang XF, Liu JL. Laparoscopic repair of inguinal hernia in adults. Ann Transl Med 2016; 4: 402.

3. Muysoms FE, Vanlander A, Ceulemans R, Kyle-Leinhase I, Michiels M, Jacobs I, Pletinckx P, Berrevoet F. A prospective, multicenter, observational study on quality of life after laparoscopic inguinal hernia repair with ProGrip laparoscopic, self-fixating mesh according to the European Registry for Abdominal Wall Hernias Quality of Life Instrument. Surg 2016; 160: 1344-1357.

4. Subwongcharoen S. Outcome of inguinal hernia repair total extraperitoneal laparoscopic hernia repair versus open tension free repair (Lichtenstein technique). J Med Assoc Thai Chotmaihet Thangphaet 2002; 85: 1100-1104.

5. Kargar S, Shiryazdi SM, Zare M, Mirshamsi MH, Ahmadi S, Neamatzadeh H. Comparison of postoperative short-term complications after laparoscopic transabdominal preperitoneal (TAPP) versus Lichtenstein tension free inguinal hernia repair: a randomized trial study. Minerva Chirurgica 2015; 70: 83-89.

6. Lichtenstein IL, Shulman AG, Amid PK, Montllor MM. The tension-free hernioplasty. Am J Surg 1989; 157: 188-193.

7. Otsuka S, Kaneoka Y, Maeda A, Takayama Y, Fukami Y, Onoe S. Ultrasonic energy device versus monopolar energy device in laparoscopic transabdominal preperitoneal (TAPP) inguinal hernia repair. Updates Surg 2016.

8. Ye Q, Chen Y, Zhan X, Zhu J. The superior inguinal ligament approach of single incision laparoscopic surgery for total extraperitoneal inguinal hernia repair. J Laparoendosc Adv Surg Tech Part A 2016.

9. Phillips EH, Arregui M, Carroll BJ, Corbitt J, Crafton WB, Fallas MJ, Filipi C, Fitzgibbons RJ, Franklin MJ, McKernan B. Incidence of complications following laparoscopic hernioplasty. Surg Endosc 1995; 9: 16-21.

10. Dion YM, Morin J. Laparoscopic inguinal herniorrhaphy. Canadian J Surg J Canadien de Chirurgie 1992; 35: 209-212.

11. Ismail M, Garg M, Rajagopal M, Garg P. Impact of closedsuction drain in preperitoneal space on the incidence of seroma formation after laparoscopic total extraperitoneal inguinal hernia repair. Surg Laparosc Endosc Percut Tech 2009; 19: 263-266.

12. Choi YY, Kim Z, Hur KY. Swelling after laparoscopic total extraperitoneal repair of inguinal hernias: review of one surgeons experience in 1,065 cases. World J Surg 2011; 35: 43-46.

13. Surgit O, Cavusoglu NT, Kilic MO, Unal Y, Kosar PN, Icen D. Use of fibrin glue in preventing pseudorecurrence after laparoscopic total extraperitoneal repair of large indirect inguinal hernia. Ann Surg Treat Res 2016; 91: 127-132.

14. Pochhammer J, Lang S, Scuffi B, Schaffer M, Smaxwil CA. Are routine ultrasound examinations helpful in the detection of bleeding complications following laparoscopic inguinal hernia repair? J Clinic Ultras JCU 2017; 45: 145-149. 
Laparoscopic inguinal hernia repair and Lichtenstein tension-free hernia repair for children in 13-18 years old: a prospective, randomized, single-blind controlled trial

15. Kapiris S, Mavromatis T, Andrikopoulos S, Georgiades C, Floros D, Diamantopoulos G. Laparoscopic transabdominal preperitoneal hernia repair (TAPP): stapling the mesh is not mandatory. J Laparoendosc Adv Surg Tech Part A 2009; 19: 419-422.

\section{*Correspondence to}

Rongpeng Zhang

Department of Pediatric Surgery

Linyi People's Hospital

Shandong University

PR China 